\title{
Two Cases of COVID-19 with Persistently Positive SARS-CoV-2- Specific IgM During One-Year Follow-Up - Sichuan Province, China, February 2021
}

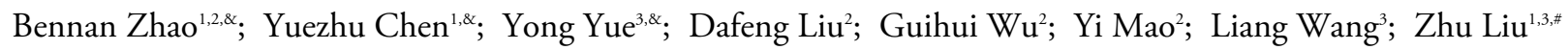

On January 22, 2020, a 41-year-old male (Patient A) developed a fever with a maximum temperature of $38{ }^{\circ} \mathrm{C}$ after returning to Chengdu City from Wuhan City. He was admitted to the Public Health Clinical Center of Chengdu on January 26, 2020, after being confirmed to be positive for coronavirus disease 2019 (COVID-19) by Chengdu CDC. Chest computed tomography (CT) revealed multiple plaque-like, ground-glass shadows in the upper, middle, and lower lobes of the right lung and upper and lower lobes of the left lung, as well as a small amount of pericardial effusion.

On January 23, 2020, a 45-year-old female (Patient B) developed shortness of breath and muscle soreness, along with decreased muscle strength after returning from Japan. After 5 days, she experienced a fever $\left(\mathrm{T}_{\max }=38.3{ }^{\circ} \mathrm{C}\right)$ and was admitted to Sichuan Provincial People's Hospital for treatment. She tested positive for COVID-19 by Chengdu CDC on January 29, 2020. Chest CT revealed plaque-like, ground-glass density shadows and nodular shadows in multiple lobes and segments, especially in the upper lobes of both lungs. About half a month later, another chest CT showed a small amount of pericardial effusion.

Patients A and B were categorized as common-type cases of COVID-19 according to the Diagnosis and Treatment Protocol for COVID-19 (Trial Version 5) (1). Laboratory tests on admission showed that the lymphocyte count, platelet count, $\mathrm{CD} 4^{+} \mathrm{T}$-cell count, and $\mathrm{CD}^{+}{ }^{+} \mathrm{T}$-cell count of Patient $\mathrm{A}$ were below the normal range. In Patient $\mathrm{B}$, only the $\mathrm{CD}^{+}{ }^{+} \mathrm{T}$-cell count was below the normal range. During follow-up, the platelet counts of Patient A were always below the normal range $\left(100 \times 10^{9} / \mathrm{L}-300 \times 10^{9} / \mathrm{L}\right)$; the lowest level was $32 \times 10^{9} / \mathrm{L}$, and the most recent result was $75 \times 10^{9} / \mathrm{L}$. At the hospital, Patient B was identified as possibly having anxiety as her most recent Depression, Anxiety, and Stress Scale-21 Items (DASS-21) total score reached 23. In both patients, pericardial effusions were observed in chest radiology images, suggesting a possibility of pericarditis. In addition, cardiac magnetic resonance imaging (MRI) indicated myocardial microcirculation injury. The physical and mental health of both patients were affected.

As of February 9, 2021, Patients A and B have been followed-up with 7 times within a year (Figure 1). Both patients tested positive for COVID-19 using nucleic acid tests at their first follow-up visit about half a month after discharge and were immediately readmitted to the hospital. After the second discharge from the hospital, both patients continued to participate in regular follow-ups, and their nucleic acid tests remained negative. However, their SARS-CoV-2specific IgM remained positive from the fourth followup visit (approximately four months after the onset of symptoms) up to the latest follow-up visit (about a year after the onset of symptoms).

IgM typically appears in the early stage and has a rather short maintenance time, so IgM is often used as an indicator for diagnosis and infection. In a study in Spain, the estimated duration of the persistence of IgM was 1.95 months, which was much shorter than the duration in our cases (2). In this study, SARS-CoV-2specific IgM was detected using chemiluminescence immunoassay (CLIA) with the i 3000 Automatic Chemiluminescence Immunoassay Analyzer (Maccura Biotechnology Co.) and the SARS-CoV-2 IgM Detection Kit (Maccura Biotechnology Co.). Among all 34 patients who were followed-up with for one year, only Patients A and B presented with persistently positive IgM. Both Patients A and B met the discharge standard but had a recurrence of positive nucleic acid test results at the first follow-up visit, and their SARSCoV-2-specific IgM remained positive for more than 8 months. In a cross-sectional study in Wuhan, $1.5 \%$ of respondents with SARS-CoV-2 infection also presented with positive $\operatorname{IgM}$ at approximately 9 months after infection (3).

In general, persistently positive $\operatorname{IgM}$ results are associated with reinfection or recurrence. In this case, there was little possibility for reinfection as these patients had no close contact with other patients infected with COVID-19 during hospitalization and 


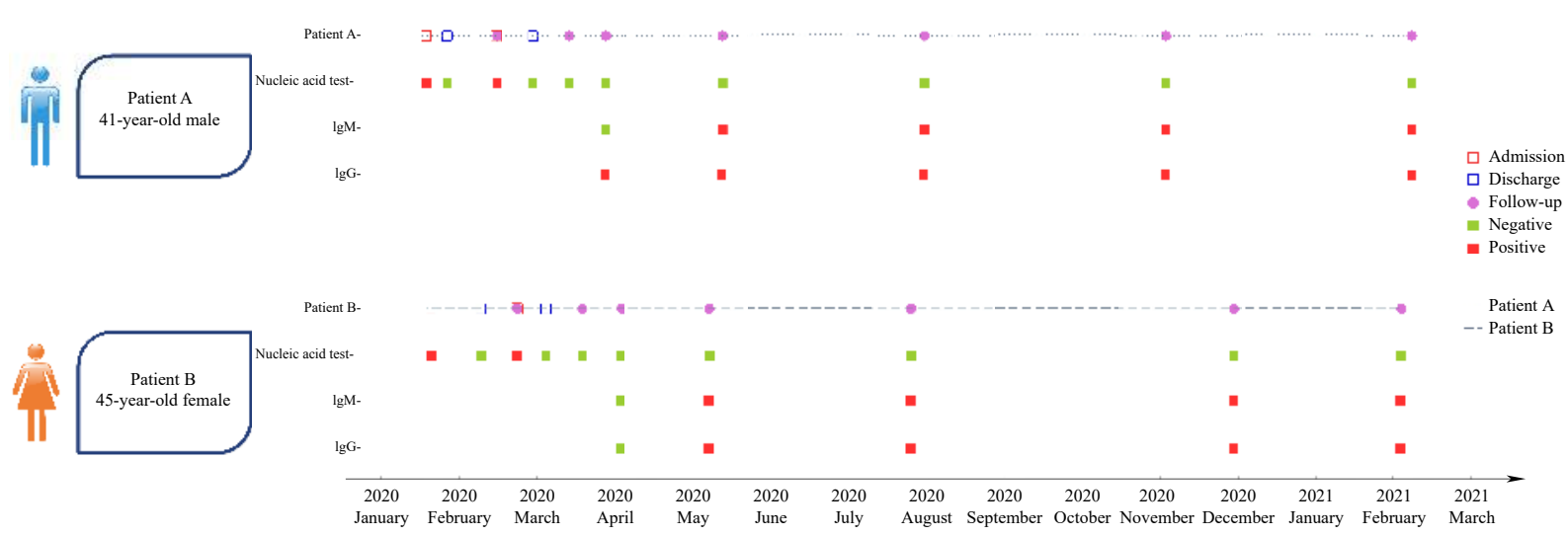

FIGURE 1. The timeline of admission, discharge, follow-up visits, and diagnostic testing for Patients A and B - China, 2020-2021.

rehabilitation. In addition, Patients $\mathrm{A}$ and $\mathrm{B}$ did not have additional recurrences since the second follow-up visit. This phenomenon may have indicated that the virus in their body was not completely eliminated. It was possible that the viral replication was maintained at a low level so that viral load was below the limit of detection. A recent study indicated that declining IgM may be a sign of virus clearance (4), which is consistent with our speculation. Despite the fact that both patients were non-severe COVID-19 cases, their CD ${ }^{+}$ $\mathrm{T}$-cell count and/or $\mathrm{CD} 8^{+} \mathrm{T}$-cell count were below the normal range during hospitalization and had no sign of significant increase during rehabilitation, even though both patients received treatment for immunomodulation. For most COVID-19 patients, including those who went through regular follow-up visits in the Public Health Clinical Center of Chengdu, $\mathrm{CD}^{+}{ }^{+} \mathrm{T}$-cell and $\mathrm{CD}^{+}{ }^{+} \mathrm{T}$-cell levels increased over time. Therefore, Patients A and B most likely had suppressed and/or dysregulated immune system activity as their immune systems may have taken longer to eliminate the virus completely. The remaining virus can stimulate the body to produce antibodies, leading to persistently positive SARS-CoV-2-specific IgM.

Even though Patients A and B did not have additional recurrences since the second follow-up visit, the results showing persistently positive SARS-CoV-2specific IgM may indicate the presence of virus and a possibility of long-term, intermittent virus shedding. This suggested that patients with persistently positive IgM results still have a risk of transmission. In this situation, close follow-ups and regular nucleic acid tests should be prioritized, and further studies are needed to identify the infectivity of patients. In addition, a potential myocardial microcirculation injury was also observed in Patients $\mathrm{A}$ and $\mathrm{B}$, so the long-term sequelae of cardiovascular damage and psychological problems should also be carefully considered in such cases.

Funding: Non-Profit Central Research Institute Fund of Chinese Academy of Medical Sciences (No.2020-PT330-005) and Chengdu Municipal Science and Technology Bureau Key R\&D Support Program Technology Innovation R\&D Project (No.2020-YF05-00133-SN).

doi: $10.46234 / \mathrm{ccdcw} 2021.172$

\# Corresponding author: Zhu Liu, 137949813@qq.com.

\footnotetext{
${ }^{1}$ Chengdu Workstation for Emerging Infectious Disease Control and Prevention, Chinese Academy of Medical Sciences, Chengdu, Sichuan, China; ${ }^{2}$ Public Health Clinical Center of Chengdu, Chengdu, Sichuan, China; ${ }^{3}$ Chengdu Center for Disease Control and Prevention, Chengdu, Sichuan, China.

\& Joint first authors.
}

Submitted: May 07, 2021; Accepted: June 09, 2021

\section{REFERENCES}

1. National Health Commission. Diagnosis and treatment protocol for COVID-19 (Trial Version 5). http://www.nhc.gov.cn/xcs/zhengcwj/ 202002/3b09b894ac9b4204a79db5b8912d4440.shtml. [2020-2-5]. (In Chinese).

2. Moncunill G, Mayor A, Santano R, Jiménez A, Vidal M, Tortajada M, et al. SARS-CoV-2 seroprevalence and antibody kinetics among health care workers in a Spanish hospital after 3 months of follow-up. J Infect Dis 2021;223(1):62 - 71. http://dx.doi.org/10.1093/infdis/jiaa696.

3. He ZY, Ren LL, Yang JT, Guo L, Feng LZ, Ma C, et al. Seroprevalence and humoral immune durability of anti-SARS-CoV-2 antibodies in Wuhan, China: a longitudinal, population-level, cross-sectional study. Lancet 2021;397(10279):1075 - 84. http://dx.doi.org/10.1016/S01406736(21)00238-5.

4. Zhou W, Xu XM, Chang ZG, Wang H, Zhong XF, Tong XL, et al. The dynamic changes of serum IgM and IgG against SARS-CoV-2 in patients with COVID-19. J Med Virol 2021;93(2):924 - 33. http://dx.doi.org/ $10.1002 /$ jmv.26353. 